\title{
Concurso de investigación en cirugía José Félix Patiño Restrepo
}

\author{
José Félix Patiño Restrepo Surgery research contest
}

\author{
Adriana Córdoba \\ MD., FACS., especialista en Cirugía General y Cirugía Laparoscópica Avanzada; profesora asociada, Fundación Universitaria \\ de Ciencias de la Salud, Hospital de San José, Bogotá, D.C., Colombia.
}

La Asociación Colombiana de Cirugía ha tenido como uno de sus objetivos primordiales el fomento y el apoyo de la investigación en cirugía, los cuales se han venido desarrollando desde su fundación, a través de las diferentes actividades, medios de comunicación y, de manera muy especial, durante el congreso nacional anual, en el que tradicionalmente se han creado los espacios para que, tanto los médicos residentes como los asociados, tengan la oportunidad de presentar y discutir sus trabajos de investigación en escenarios de alta calidad científica y académica, en los que participan especialistas nacionales y extranjeros.

Fue así como en marzo de 1993, siendo el doctor Jaime de la Hoz el presidente de nuestra Asociación, se llevó a cabo por primera vez lo que se bautizó con el nombre de Simposio Nacional del Residente Quirúrgico. Su objetivo fue la creación de un escenario, inmerso en las actividades del congreso anual de la Asociación, para que los residentes de cirugía tuvieran la oportunidad de presentar sus trabajos de investigación, los cuales serían seleccionados, discutidos, evaluados y premiados por cirujanos y profesores, en el marco de una atmósfera académica y científica.

Desde entonces, este Simposio se ha llevado a cabo de manera ininterrumpida hasta el día de hoy, con una dinámica orientada, no sólo hacia el estímulo de la investigación por parte los residentes de cirugía, sino también hacia la mejora progresiva de la calidad de los trabajos. En ese orden de ideas, se establecieron procesos formales para la selección inicial de los trabajos, que deben cumplir con requisitos básicos de pertinencia, calidad y metodología para ser aceptados, para su presentación y posterior evaluación por parte de jurados calificadores, integrados por especialistas nacionales e invitados internacionales.

Palabras clave: cirugía general; programas de postgrado; investigación; academias e institutos; grupos de investigación; evaluación de la investigación en salud.

Keywords: general surgery; health postgraduate programs; research; academies and institutes; research groups; health research evaluation.

Fecha de recibido: 15/11/2020 - Fecha de aceptación: 24/02/2021 - Fecha de publicación en línea: 05/03/2021

Correspondencia: Adriana Córdoba, Calle 10 \# 18-75, oficina de Cirugía general, Hospital de San José, Bogotá, D.C., Colombia.

Teléfono: 3538000 Extensión 185, 3153451154. Correo electrónico: apcordoba@hospitaldesanjose.org.co cordobadriana@gmail.com Citar como: Córdoba A. Concurso de investigación en cirugía José Félix Patiño Restrepo. Rev Colomb Cir. 2021;36:183-4. https://doi.org/10.30944/20117582.911

Este es un artículo de acceso abierto bajo una Licencia Creative Commons - BY-NC-ND https://creativecommons.org/licenses/by-ncnd/4.0/deed.es 
Los mejores trabajos son premiados en la sesión solemne de clausura del congreso y posteriormente publicados en nuestra Revista Colombiana de Cirugía.

Con el fin de fortalecer la investigación en cirugía como actividad fundamental en la formación y en la vida de nuestros cirujanos, hemos querido honrar la memoria de una de las figuras más sobresalientes de la Medicina y la Cirugía colombianas, maestro por excelencia, científico y cirujano por vocación, autor e investigador prolífico, pionero y fundador de nuestra Asociación, cuya sabiduría y enseñanzas tuvimos la fortuna de haber compartido muchos de nosotros: el doctor José Félix Patiño Restrepo. En su honor hemos tomado la decisión de modificar el nombre de Simposio Nacional del Residente Quirúrgico, que en lo sucesivo se llamará Concurso de investigación en cirugía José Félix Patiño Restrepo.

Para el 2021 hemos introducido algunos cambios en la dinámica de nuestro concurso, orientados a extender la participación, tanto de los médicos residentes como de los especialistas, para lo que se establecerán diferentes categorías. Desde ahora queremos invitarlos a que envíen sus trabajos y participen en nuestro concurso y de esta manera contribuyan al fortalecimiento de la investigación, considerada hoy día una herramienta fundamental para el ejercicio científico y académico de nuestra profesión. 